\title{
N_LyST: a simple and rapid screening test for Lynch Syndrome
}

Susanti Susanti ${ }^{1}, 2,3^{*}$, Wakkas Fadhil ${ }^{1,3^{*}}$, Henry O. Ebili ${ }^{1,3,4^{*}}$, Asiri Abutaleb ${ }^{1,3}$, Ausrine Nestarenkaite ${ }^{5}$, Efthymios Hadjimichael ${ }^{1,3}$ Hersh A. Ham-Karim ${ }^{1,3}$, Joanne Field ${ }^{6}$, Katherine Stafford ${ }^{6}$, Balawir Matharoo-Ball ${ }^{7}$, James C.Hassall1,3, Abid Sharif 6 , Anca Oniscu ${ }^{8}$, Mohammad llyas ${ }^{1,3}$

${ }^{*}$ Co-first authors

${ }^{1}$ Molecular Pathology Group, Unit of Academic Molecular Pathology, Division of Cancer and Stem Cell, School of Medicine, University of Nottingham, Queen's Medical Centre Campus, The University of Nottingham, Nottingham, UK. 2Deparment of Pharmacology and Clinical Pharmacy, Faculty of Pharmacy, University of Muhammadiyah Purwokerto, Central Java, Indonesia. ${ }^{3}$ Nottingham Molecular Pathology Node, University of Nottingham, Queen's Medical Centre Campus, The University of Nottingham, Nottingham, UK. ${ }^{4}$ Department of Morbid Anatomy and Histopathology, Olabisi Onabanjo University, Ago-Iwoye, Nigeria.

${ }^{5}$ National Center of Pathology, Affiliate of Vilnius University Hospital Santaros Klinikos, Lithuania. ${ }^{5}$ East Midlands Regional Molecular Genetics Service, Nottingham University Hospitals NHS Trust, Nottingham, UK. ${ }^{7}$ Nottingham Health Sciences Biobank, Nottingham University Hospitals NHS Trust, Nottingham, UK. ${ }^{8}$ Molecular Pathology, Royal Infirmary of Edinburgh, 51 Little France Crescent, Edinburgh. EH16 4SA.

Corresponding author: Prof M llyas

\section{Running Title: N_LyST}

Word count: 3796

Keywords: Microsatellite Instability (MSI), High Resolution Melting (HRM), Colorectal cancer, Lynch Syndrome, BRAF mutation, promoter methylation 


\section{ABSTRACT}

\section{Background and aims}

We sought to use PCR followed by high-resolution melting (HRM) analysis to develop a single closed-tube screening panel to screen for Lynch Syndrome. This comprises tests for microsatellite instability (MSI), MLH1 methylation promoter and $B R A F$ mutation.

\section{Methods}

For MSI-testing, 5 mononucleotide markers (BAT25, BAT26, BCAT25, MYB, EWSR1) were developed. In addition, primers were designed to interrogate Region $\mathrm{C}$ of the MLH1 promoter for methylation (using bisulphite-modified DNA) and to test for mutations in codon 600 of BRAF. Two separate cohorts from Nottingham $(n=99$, 46 with MSI, 53 being microsatellite stable (MSS)) and Edinburgh ( $n=88,45$ MSI, 43 MSS).

\section{Results}

All the cases $(n=187)$ were blind tested for MSI and all were correctly characterised by our panel. The $M L H 1$ promoter and BRAF were tested only in the Nottingham cohort. Successful blinded analysis was performed on the MLH1 promoter in 97 cases. All MSS cases showed a pattern of non-methylation whilst 41/44 cases with MSI showed full methylation. The three cases with $\mathrm{MSI}$ and a non-methylated pattern had aberrations in $\mathrm{MSH} 2$ and $\mathrm{MSH} 6$ expression. BRAF mutation was detected in $61 \%$ of MSI cases and $11 \%$ of MSS cases.

Finally, 12 cases were blind screened by using the whole panel as a single test. Of these, 5 were identified as MSS, 4 as MSI/non-LS and 3 as MSI/possible LS. These results were concordant with the previous data.

\section{Conclusion}

We describe the №ttingham Lynch Syndrome Iest (N_LyST). This is a quick simple cheap method for screening for Lynch Syndrome. 


\section{INTRODUCTION}

Colorectal cancer (CRC) is a leading causes of cancer-related mortality (1-3). Most CRCs arise sporadically without any antecedent family history. There are, however, several cancer syndromes in which development of CRC is part of the phenotypic spectrum $(1,2)$. The most common of these is Lynch Syndrome (also known as Hereditary Non-Polyposis Colorectal Cancer) which is responsible for $2-4 \%$ of all CRCs $(3,4)$. Patients with LS are susceptible to the development of CRCs and to the development of extra-colonic tumours - most notably endometrial, ovarian and small intestine adenocarcinomas $(5,6)$

Lynch Syndrome (LS) arises as a consequence of germline mutation in one of four DNA mismatch repair (MMR) genes (i.e. MLH1, PMS2, MSH2, MSH6) (7, 8). Loss of any of the proteins results in loss of MMR function and an increase in the rate of gene mutation. One of the manifestations of this is an increase in insertion-deletion (indel) mutations especially at DNA microsatellites - known as microsatellite instability (MSI) $(8,9)$. Tumours arising in LS therefore usually show both loss of expression of at least one of the MMR proteins (i.e. dMMR) and MSI $(10,11)$. Thus, $\mathrm{dMMR}$ and MSI - although they are distinct phenomena, are usually regarded as synonymous. Conversely, proficient mismatch repair (pMMR) is considered synonymous with a microsatellite stable (MSS) phenotype.

Numerous studies have shown that, due to the high risk of multiple cancers and its relatively high prevalence, there is a clinical and economic benefit to be gained by screening CRCs for LS (12-15). Whilst a definitive diagnosis of LS can only be made by demonstration of a germline mutation in an MMR gene, the possibility of LS can be inferred if a tumour is shown to be dMMR or shown to have MSI. However, approximately $10-15 \%$ of sporadic CRCs will also show dMMR/MSI due to somatic loss of MMR function (10). Epigenetic silencing of the MLH1 gene is the most common cause of dMMR in sporadic tumours and very rarely occurs in LS $(16,17)$. Thus sporadic tumours with $\mathrm{dMMR} / \mathrm{MSI}$ can be distinguished from tumours arising in LS by demonstrating methylation of the MLH1 promoter. Similarly, somatic mutation of $B R A F$ is common in sporadic tumours with $\mathrm{MSI}$ but very rarely occurs in tumours arising in LS (17-19).

Guidance from the National Institute of Clinical and Healthcare Excellence (NICE) recommends that all CRCs should be screened for the possibility of LS (12). The pathway suggested involves two steps: firstly, identify cases with dMMR/MSI and 
then filter out sporadic cases by testing for BRAF mutation and $M L H 1$ promoter methylation. For the first step, testing for $d M M R$ can be performed by immunohistochemistry $(\mathrm{IHC})$ whilst testing for $\mathrm{MSI}$ involves $\mathrm{PCR}$ followed by capillary electrophoresis. For the second step, PCR followed by mutation screening or sequencing is required for detection of BRAF mutation. Testing for MLH1 promoter methylation can be performed by PCR on modified DNA followed by sequencing or gel electrophoresis.

This strategy uses multiple tests and requires downstream analysis of the PCR products on different platforms. We believed that testing could be simplified using High Resolution Melting (HRM) analysis. HRM is an exquisitely sensitive method for detecting variations in DNA sequence (20-22). It can be performed at the end of a PCR without needing to transfer PCR products to another tube (i.e. a closed-tube test). We have shown previously that HRM can be used for testing for microsatellite instability $(23,24)$, for detection of BRAF mutation (23-27) and to identify promoter methylation (25). Here we sought to create a single panel test in which a single PCR run followed by HRM can be used to screen for patients at risk of Lynch Syndrome.

\section{MATERIALS AND METHODS}

\section{Cell lines}

CRC cell lines were kindly donated by Prof lan Tomlinson. The cell lines DLD1, HCT116, RKO, LoVo, and LS1034 have previously been shown to have MSI whilst the cell lines SW480, SW620, HUTU80, SW837 have been shown to be microsatellite stable (MSS) (28). DNA was extracted from cell lines using the Qiagen DNeasy kit (Qiagen, UK) as per manufacturer's instructions and adjusted to a concentration of $20 \mathrm{ng} / \mu \mathrm{l}$. Identity of the cell lines was confirmed by mutation profiling as previously described (25).

Two diploid cell lines were chosen for spiking experiments in order to perform limit of detection experiment. DNA extracted from HCT116 (an MSI cell line) was spiked into DNA extracted from SW837 (MSS), to produce mixtures of DNA containing various proportion of $\mathrm{HCT} 116$ of $\approx 50 \% ; \approx 25 \% ; \approx 12.5 \% ; \approx 6 \% ; \approx 3 \%$ and $\approx 1.5 \%$.

\section{Primary colorectal cancers}

The Nottingham cohort 
Ninety nine cases of CRC, which had previously been tested by immunohistochemistry for expression of MMR proteins, were retrieved from the archives of Nottingham University Hospital (NUH) Pathology Department. Of these, 46 cases which were dMMR (and by inference had MSI). The remaining 53 cases were pMMR (and by inference were MSS). Access to tissues and ethics approval were granted by Nottingham Health Sciences Biobank which has approval as an IRB from North West - Greater Manchester Central Research Ethics CommitteeREC reference: $15 / \mathrm{NW} / 0685$.

\section{The Edinburgh cohort}

Eighty eight cases of CRC were retrieved form the archives of the Royal Infirmary of Edinburgh, Pathology Department which had previously been tested for expression of MMR proteins or MSI. Of these, 45 cases were dMMR/MSI and 43 cases were pMMR/MSS. Access to annonymised use of tissues was granted by Tissue Governance NHS Lothian under ethics approval number SR783.

\section{DNA extraction for formalin-fixed tissue}

DNA was also extracted from formalin-fixed paraffin-embedded (FFPE) tumour samples. One or two $20 \mu \mathrm{m}$ thick sections (depending on tissue surface area) were cut from each block. DNA was extracted using the QIAamp DNA FFPE tissue kit (Qiagen Ltd, UK) following the manufacturer's protocol. All DNA samples were adjusted to a concentration of $20 \mathrm{ng} / \mu \mathrm{l}$.

\section{Validation of immunohistochemistry as a marker of MMR deficiency}

The Nottingham cohort had been tested for expression of the MMR proteins by immunohistochemistry (IHC). In order to confirm that the interpretation of the $\mathrm{IHC}$ was a correct reflection of the MMR function, a group of 33 cases (15 MSI/18 MSS) were tested by PCR followed by capillary electrophoresis (CE). PCR and CE testing was performed by the Molecular Genetics Laboratory at Nottingham University Hospitals NHS trust using the Promega MSI System version 1.2 in accordance with manufacturer's instructions. Five mononucleotide markers for MSI testing (BAT-25, BAT-26, NR-21, NR-24, MONO-27) (29) and two pentanucleotide markers (Penta-D, Penta-E) for sample identity checking, were amplified using fluorescently-labelled primers in a multiplex PCR. Products were analysed by capillary electrophoresis on 
an Applied Biosystems 3130xl Genetic Analyzer (Life Technologies) using the kit internal lane standard. Data were analysed using GeneMapper® software. Samples that showed microsatellite instability at $\geq 2$ mononucleotide loci were interpreted as having MSI.

\section{Identification of novel markers for MSI testing}

A total of 11 different mononucleotide repeat microsatellite loci with potential utility as sensitive markers of MSI were tested. Of these, two markers (BAT 25 and BAT 26) are established $\mathrm{MSI}$ markers although the primers for these loci were redesigned (30-33). Two markers (BCAT 25 and TYMS 26) were identified via bioinformatics analyses by our group (we have previously described BCAT $25(23,24)$ as a useful marker), two markers (EWSR1 and MONO-27) were identified from published research articles (34-36) and five markers (MYB, ANGEL2, TP53 (BAT34CA), FBXO46, and TCF4) were identified in the SelTar database (37) (www.seltarbase.org). The individual markers were chosen if (i) the mononucleotide repeat size is $>10$ bases and (ii) if the published rate of mutation of the marker in $\mathrm{MSI}$ tumours (CRC and gastric cancer) is $>80 \%$. Primers were designed using a combination of online design tools: MFEprimers (http://mfeprimer.igenetech.com), UCSC in silico PCR (http://rohsdb.cmb.usc.edu/GBshape/cgi-bin/hgPcr.) and Reverse Complement (www.bioinformatics.org.).

The optimum annealing temperatures of the primer pairs was ascertained as previously described (38). Supplementary Table 1 lists all the mononucleotide repeat microsatellite markers, their genomic locations, amplicon sizes, the lengths of the mononucleotide repeats and the ranges of optimum annealing temperature. $A$ range of metrics were used in order to define the best primers including reproducibility, PCR efficiency and range of functioning annealing temperature.

\section{Testing for MSI using HRM analysis}

In order to test for MSI using HRM, PCR was carried out each sample on the ABI 7500 FAST Real-Time PCR System (Applied Biosystems). Each reaction was carried out in a final volume of $10 \mu \mathrm{l}$ and contained $5 \mu \mathrm{l}$ of $2 \mathrm{x}$ Hot Shot Diamond PCR master mix, $0.5 \mu$ l of $20 x(25 \mu \mathrm{M})$ EvaGreen dye, each primer final concentration was at $0.25 \mu \mathrm{M}$ and $20 \mathrm{ng}$ DNA template. The PCR was performed using a 3-step procedure: 1 cycle of $\left(95^{\circ} \mathrm{C} / 5\right.$ minutes $), 45$ cycles of $\left[\left(95^{\circ} \mathrm{C} / 10\right.\right.$ seconds $) \mathrm{X} 1 ;\left(55^{\circ} \mathrm{C} / 30\right.$ 
seconds)X1; $\left.\left(72^{\circ} \mathrm{C} / 30\right) \mathrm{X} 1\right]$ and 1 cycle of $\left(72^{\circ} \mathrm{C} / 2\right.$ minutes $)$. HRM was performed intube immediately after PCR and consisted of heating to $95^{\circ} \mathrm{C}$ for 15 seconds, rapid cooling to $60^{\circ} \mathrm{C}$ and maintenance at $60^{\circ} \mathrm{C}$ for 1 minute. This was followed by slow ramping up at 0.03 degrees $/ \mathrm{s}$ to $95^{\circ} \mathrm{C}$ during which fluorescent data were captured. The melting data were analysed following normalisation but without temperature shifting using the $A B I$ HRM software v2.0. Samples were regarded as $M S I$ if $\geq 2$ markers (40\%) showed instability; otherwise, they were regarded as microsatellite stable (MSS) tumours.

The limit of detection for MSI by both CE and HRM was tested using spiked DNA samples (as described above).

\section{Novel primers for BRAF testing}

We have previously designed primers for screening for BRAF mutation using the nested QMC-PCR protocol $(26,27)$. For the purposes of this protocol, which requires a single stage PCR, novel primers were designed specifically for detection of mutation at codon 600 .

\section{Testing for methylation of the MLH1 promoter}

\section{Primer design}

Bisulphite modification of DNA causes a conversion of non-methylated cytosine residues to uracil whilst the methyl group of the methylated cytosines protects against this change (and cytosines are preserved). Following PCR on bisulphite modified DNA, the methylated cytosines remain whilst non-methylated cytosines are converted to thymine residues. The sequence of methylated / non-methylated DNA is therefore different and can be discriminated by HRM.

The promoter of $M L H 1$ contains $4 \mathrm{CpG}$ rich regions (labelled A - D) which are the targets of epigenetic modification. It is generally considered that hypermethylation of the $\mathrm{CpG}$ island in Region C is related to MLH1 silencing (39). Furthermore, it is reported that Region $\mathrm{C}$ exists in a dichotomous state i.e. all $\mathrm{CpG}$ residues being either methylated or non-methylated $(39,40)$ without a state of partial methylation. However, the exact location of Region $C$ is not well defined and the number of reported CpG residues varies between 5 and $8(39,40)$. Using the publicly available data, we identified a part of Region $C$ (located -46 to -111 from the transcription start site; NCBI sequence ID: NC_018914.2) which would contain all 8 of the 
reported methylated $\mathrm{CpG}$ residues (Supplementary Figure 1). Primers were designed to interrogate the whole $\mathrm{CpG}$ island of Region $\mathrm{C}$ using the exactly the same cycling and HRM parameters as for the MSI markers. All tests (both sequencing and HRM) for MLH1 Region C promoter methylation were performed on bisulphite modified DNA.

\section{Bisulphite conversion of DNA}

In order to test for methylation of the MLH1 promoter, it was necessary to modify the DNA. Bisulfite conversion of $400 \mathrm{ng}$ of genomic DNA from each sample was carried out using the EZ-DNA Methylation-Lightning ${ }^{\mathrm{TM}}$ Kit (Zymo Research, USA), according to the manufacturer's protocol. Optimisation of the methylation detection HRM-PCR assay was carried out using completely methylated or non-methylated human control DNA (Qiagen Ltd, UK).

\section{Sequencing of Region C of the MLH1 promoter}

In order to confirm the dichotomous methylation state of Region C, twenty CRCs (10 pMMR, $10 \mathrm{dMMR}$ ) were selected from the Nottingham cohort for Sanger sequencing of modified DNA. PCR prior to sequencing was performed using the reverse primer as described above. The forward primer however was modified to include a "squirrel" tail to allow sequencing of short fragments as previously described (38). PCR products were purified using the QIAquick kit (Qiagen) and the products sent to the DNA sequencing facilities (School of Life Sciences, University of Nottingham) and sequenced using Applied Biosystems BigDye Terminator v3.1 Cycle Sequencing Kit and 3130xI ABI PRISM Genetic Analyzer (Data collection software v3.0, Sequence analysis software v5.2). The chromatograms were interpreted using Finch TV 1.4.0 free software from www.geospiza.com/finchtv .

\section{Evaluation of the N_LyST panel}

All the biomarkers were tested together as a single panel test. Twelve cases were selected from the Nottingham cohort. This selection contained five cases designated as MSS, four cases designated as MSI with MLH1 deficiency and three cases designated as MSI with deficiency of MSH2/MSH6. They were assigned a new ID and were tested blind. 


\section{Statistical analysis}

GraphPad Prism software version 5.0 was used for statistical analysis. The Chi squared test was used to test for association between different factors. A value of $p<0.05$ was taken as being statistically significant.

\section{RESULTS}

\section{Validation of IHC as a marker of mismatch repair function}

The Nottingham cohort had been selected using IHC expression of MMR proteins as a marker of MMR function. To confirm the association between IHC data and the presence or MSI, 33 cases from this cohort were tested by CE for the presence of MSI. Of these, 15 had been designated dMMR and 18 were pMMR. There was $100 \%$ concordance between the IHC analysis and MSI test results.

\section{Utility of HRM for detection of MSI}

From 11 different potential microsatellite loci, a panel of 5 markers comprising BAT25, BAT26, BCAT25, MYB and EWSR1 was chosen as the one showing the best performance. Our panel was compared with the commercial CE panel for their limit of detection for $\mathrm{MSI}$ calling. Using spiked samples containing varying proportions of DNA from MSI/MSS cell lines, the CE method and HRM were comparable with a limit of detection $\approx 6.25 \%$ (Figure 1).

Our panel was used to test the Nottingham cohort of 99 cases of CRC (46 dMMR and 53 pMMR) and both observers correctly called every case. The Edinburgh cohort of 88 (45 dMMR/MSI and 43 pMMR/MSS) were tested separately. The HRM data were analysed by the same two observers and one observer correctly called all cases whilst the other observer miscalled 2 case of MSI as MSS. Although we applied the generally used threshold of instability at $\geq 2$ markers (40\%) for a call of MSI, most cases usually showed instability at 4-5 markers and only one case, out of the total of 91 cases designated $\mathrm{dMMR} / \mathrm{MSI}$, was found to have instability at only 2 markers. Of the cases designated as MSS, 7\% (7/96) had instability at 1 marker only whilst the remainder did not show any alteration in the microsatellite markers.

\section{Screening for $B R A F$ mutation}

New primers to screen for BRAF codon 600 mutation were designed and optimised to work as a single stage test using the cycling conditions for MSI testing. Primers 
were optimised and tested on cell lines with known BRAF mutation status (data not shown). All cases in the Nottingham cohort were tested and 28/46 (61\%) of cases designated as MSI showed mutation whilst 6/53 (11\%) of the MSS cases showed mutation. This frequency of mutation is consistent with published data and confirm the significant association of MSI with $B R A F$ mutation (chi squared test, $\mathrm{p}<0.0001$ ).

\section{Analysis of $M L H 1$ promoter methylation}

\section{Sequencing of Region $C$}

Twenty cases of CRC form the Nottingham cohort (10 MSI, $10 \mathrm{MSS}$ ) were tested for MLH1 promoter methylation by direct sequencing. Our findings replicated published data with 10/10 case of MSS CRC showing conversion of all 8 of the cytosines at the $\mathrm{CpG}$ sites to thymine without any cases suggesting partial methylation (i.e. methylation at some residues but not others). In contrast, 10/10 cases of the MSI CRCs showed retention of the cytosines at the CpG sites (Figure 2). The MSI samples did however show a double signal at the CpG sites i.e. a cytosine and a thymine. Since tumour samples contain both tumour epithelium and stroma, it is expected that the methylated signal comes from the tumour cells whilst the signal from the stroma would be non-methylated.

\section{HRM analysis of Region C}

HRM was performed following PCR with primers targeted to amplify around the CpG island of Region $\mathrm{C}$ of the MLH1 promoter. Amplification, following bisulphite modification, of both fully methylated and fully non-methylated DNA gave a single peak (Figure 3A). The melting temperature $(\mathrm{Tm})$ of the PCR product from the nonmethylated DNA (i.e. the "non-methylated peak") was lower than that of PCR product from the methylated DNA (the "methylated peak") reflecting the enrichment the latter with cytosine residues within the methylated sequence.

All cases in the Nottingham cohort were tested for methylation of Region C. Two cases (both designated as MSI) could not be tested due to failed PCR post bisulphite modification of DNA. Of the 97 successfully tested cases, two distinct melting patterns were seen i.e. a single peak low Tm peak (corresponding to the nonmethylated peak) and a double peak with both low and high Tm (corresponding to both the non-methylated peak and the methylated peak, Figure 3B). All cases 
designated as pMMR/MSS showed only a single non-methylated peak i.e. there was no promoter methylation. We regard this as the "non-methylated pattern". Of the 44 cases with MSI, 41 showed a double peak indicating both methylated DNA and nonmethylated DNA. The double peak was associated with loss of MLH1 expression (Chi squared test $\mathrm{p}<0.0001$ ) and we regard this as the "methylated pattern". The double peak is mostly likely due to methylated DNA being present in the tumour epithelium whilst the stromal cells are likely to contain non-methylated DNA. The three remaining $\mathrm{MSI}$ cases showed a single non-methylated peak. These cases were deficient in MSH2 and MSH6.

\section{Screening for Lynch Syndrome using N_LyST}

In order to test the N_LyST panel, 12 cases were blind-tested in a single PCR run. The outcome of N_LyST is to categorise cases "probable Lynch Syndrome" if they show MSI, have wild-type BRAF and have a non-methylated pattern for Region $\mathrm{C}$ of the MLH1 promoter. Any other pattern would be categorised as "not Lynch Syndrome". All cases were correctly identified by the panel (Table 1, Figure 4).

\section{DISCUSSION}

In this paper we have described the №ttingham Lynch $\underline{\text { Syndrome }}$ Test (N_LyST) as a single panel closed-tube test for Lynch Syndrome screening. The cases used to develop this test were selected on the basis of MMR protein expression and, to validate the use of these cohorts for our assay, we firstly confirmed that dMMR based on IHC was very strongly correlated with MSI.

N_LyST incorporates the three components of LS screening (i.e. testing for MSI, MLH1 promoter methylation and BRAF mutation) into a single PCR run. Firstly we developed a panel of five microsatellite markers which includes two established markers (BAT25, BAT26) and three novel markers (BCAT25, MYB and EWSR1). When tested in 187 CRCs (from two different institutions), there was near perfect concordance with the IHC/CE designation. Analysis of the HRM data was undertaken by two observers thereby demonstrating that the analysis is easy and reproducible. The HRM method has a similar limit of detection as CE analysis ( $\approx 6.25 \%$ mutant DNA) but CE analysis can be complicated by stutter bands that can cause difficulty in allele sizing $(33,41)$. 
Next we designed an assay to detect mutations in codon 600 of BRAF. Reassuringly the detected mutation frequencies (61\% in MSI tumours, $11 \%$ in MSS tumours) were in the expected range and the association of $\mathrm{MSI}$ with $B R A F$ mutation (Chi squared test, $p<0.0001$ ) was seen.

The third step was the design of an assay to test for methylation of Region $C$ of the MLH1 promoter. Our sequencing and HRM data confirmed the dichotomous state of Region C i.e. either non-methylated or fully methylated. The HRM assay clearly discriminated the two states and, when tested on the Nottingham cohort, all dMMR cases with loss of MLH1 expression by IHC had MLH1 promoter methylation (i.e. the methylated pattern of two peaks) and were therefore sporadic tumours. None of the cases which were pMMR or dMMR due to MSH2/MSH6 loss, had MLH1 promoter methylation. Finally, all components of N-LyST were put together and tested as a panel. Twelve cases of CRC were blind tested and perfectly categorised as "non-LS" or "probable LS".

N_LyST involves a panel of seven PCRs which are performed in a single run using a single cycling program. It could hugely improve workflow in a diagnostic lab since HRM is performed in-tube on completion of the PCR and transfer of PCR products to another platform for further analysis is not required. Since the test involves a panel applied to all tumours, it does mean that some tumours which are MSS will be unnecessarily tested for BRAF mutation and $M L H 1$ promoter methylation. However, the cost of this is more than off-set by savings made on manpower and consumable due to the removal of downstream analyses of PCR products. Furthermore, since it is a closed-tube test, the risk of laboratory contamination with PCR products is eliminated.

Most modern Real-Time PCR machines will have HRM capabilities and expensive specialist equipment is not required for N_LyST. The ease of the methodology and data interpretation mean the N-LyST could probably be performed in non-specialist diagnostic pathology labs. This becomes pertinent when considering that MSI testing is likely to increase as it provides information which extends beyond Lynch Syndrome testing e.g. MSI can be used to stratify patients in to groups eligible for treatment with 5-Fluorouracil based therapy $(42,43)$ or immunotherapy $(44,45)$. Such high throughput analysis will require a rapid and simple test such as N_LyST. An important question is whether N_LyST - since it is a screening test - is relevant in the era of Next Generation Sequencing (NGS). The sheer sequencing power of NGS 
platforms would allow the MMR genes and multiple microsatellites to be sequenced in a single test (46). However, microsatellites regions can be problematic from some NGS platforms and, where there is low tumour epithelium content, great sequencing depth may be required. In addition, $M L H 1$ promoter methylation testing would require Methyl-Seq to be performed. The economic analyses performed as part of the NICE guidelines concluded that it was more cost-effective to screen the tumour samples prior to germline sequencing (12). Since N_LyST can be performed in less time than that required for library preparation and sequencing with NGS, a case for including N_LyST in the testing pathway can be made.

In summary, N_LyST is based on PCR and HRM and uses a panel of 7 markers to test for MSI, MLH1 promoter methylation and BRAF mutation in a single PCR run. It can be performed on most Real-time PCR machines and, as a closed-tube test, it can improve laboratory workflow and reduce turnaround times for testing. It is a robust test which represents a quick, cheap and easy way to screen for Lynch Syndrome.

\section{Acknowledgements}

The authors acknowledge financial support from the EC Marie Curie Actions, AIDPATH project (Contract No.612471). We are very grateful to Dr S Richman for review and comments on the document. 


\section{REFERENCES}

1. Gryfe R. Inherited Colorectal Cancer Syndromes. Clinics in Colon and Rectal Surgery. 2009 Nov;22(4):198-208. PubMed PMID: 21037810.

2. Tomlinson I. The Mendelian colorectal cancer syndromes. Annals of clinical biochemistry. 2015 Nov;52(Pt 6):690-2. PubMed PMID: 26169059. Pubmed Central PMCID: PMC4611988. Epub 2015/07/15. eng.

3. Kastrinos F, Syngal S. Inherited Colorectal Cancer Syndromes. Cancer journal (Sudbury, Mass). 2011;17(6):405-15. PubMed PMID: PMC3240819.

4. Hampel H. Genetic testing for hereditary colorectal cancer. Surg Oncol Clin N Am. 2009 2009/10//;18(4):687-703. PubMed PMID: 19793575. eng.

5. Cottrell S, Bodmer WF, Bicknell D, Kaklamanis L. Molecular analysis of APC mutations in familial adenomatous polyposis and sporadic colon carcinomas. The Lancet. 1992 1992/09/12/;340(8820):626-30.

6. Ilyas M, Straub J, Tomlinson IPM, Bodmer WF. Genetic pathways in colorectal and other cancers1Reprinted from Eur J Cancer 1999, 35(3), 335-351. Please use this reference when citing this article.1. European Journal of Cancer. 1999 1999/12/01/;35(14):1986-2002.

7. Lynch HT, de la Chapelle A. Hereditary colorectal cancer. The New England journal of medicine. 2003 Mar 06;348(10):919-32. PubMed PMID: 12621137. Epub 2003/03/07. eng.

8. Peltomäki P. Role of DNA Mismatch Repair Defects in the Pathogenesis of Human Cancer. Journal of Clinical Oncology. 2003 March 15, 2003;21(6):1174-9.

9. Boland CR, Goel A. Microsatellite Instability in Colorectal Cancer. Gastroenterology. 2010;138(6):2073-87.e3. PubMed PMID: PMC3037515.

10. Chiaravalli AM, Furlan D, Facco C, Tibiletti MG, Dionigi A, Casati B, et al. Immunohistochemical pattern of hMSH2/hMLH1 in familial and sporadic colorectal, gastric, endometrial and ovarian carcinomas with instability in microsatellite sequences. Virchows Archiv. 2001 2001/01/01;438(1):39-48.

11. Shia J, Klimstra DS, Nafa K, Offit K, Guillem JG, Markowitz AJ, et al. Value of Immunohistochemical Detection of DNA Mismatch Repair Proteins in Predicting Germline Mutation in Hereditary Colorectal Neoplasms. The American Journal of Surgical Pathology. 2005;29(1):96-104. PubMed PMID: 00000478-20050100000010. 
12. Molecular Testing Strategies for Lynch Syndrome in People with Colorectal Cancer Available from: https://www.nice.org.uk/guidance/dg27/chapter/1Recommendations.

13. Grosse SD, Palomaki GE, Mvundura M, Hampel H. The cost-effectiveness of routine testing for Lynch syndrome in newly diagnosed patients with colorectal cancer in the United States: corrected estimates. Genetics in medicine : official journal of the American College of Medical Genetics. 2015 Jun;17(6):510-1. PubMed PMID: 26035801. Pubmed Central PMCID: PMC4511954. Epub 2015/06/04. eng.

14. Leenen $\mathrm{CH}$, Goverde A, de Bekker-Grob EW, Wagner A, van Lier MG, Spaander MC, et al. Cost-effectiveness of routine screening for Lynch syndrome in colorectal cancer patients up to 70 years of age. Genetics in medicine : official journal of the American College of Medical Genetics. 2016 Oct;18(10):966-73. PubMed PMID: 26938782. Epub 2016/03/05. eng.

15. Snowsill T, Coelho H, Huxley N, Jones-Hughes T, Briscoe S, Frayling IM, et al. Molecular testing for Lynch syndrome in people with colorectal cancer: systematic reviews and economic evaluation. Health technology assessment (Winchester, England). 2017 Sep;21(51):1-238. PubMed PMID: 28895526. Pubmed Central PMCID: PMC5611555. Epub 2017/09/13. eng.

16. Poynter JN, Siegmund KD, Weisenberger DJ, Long TI, Thibodeau SN, Lindor $\mathrm{N}$, et al. Molecular Characterization of MSI-H Colorectal Cancer by \&lt;em\&gt;MLHI\&lt;/em\&gt; Promoter Methylation, Immunohistochemistry, and Mismatch Repair Germline Mutation Screening. Cancer Epidemiology Biomarkers \&amp;amp; Prevention. 2008;17(11):3208.

17. Bouzourene H, Hutter P, Losi L, Martin P, Benhattar J. Selection of patients with germline MLH1 mutated Lynch syndrome by determination of MLH1 methylation and BRAF mutation. Familial Cancer. 2010 2010/06/01;9(2):167-72.

18. Deng G, Bell I, Crawley S, Gum J, Terdiman JP, Allen BA, et al. $<$ strong $><$ em $>\mathrm{BRAF}</$ em $><$ /strong $>$ Mutation Is Frequently Present in Sporadic Colorectal Cancer with Methylated hMLH1, But Not in Hereditary Nonpolyposis Colorectal Cancer. Clinical Cancer Research. 2004;10(1):191-5.

19. Thiel A, Heinonen M, Kantonen J, Gylling A, Lahtinen L, Korhonen M, et al. BRAF mutation in sporadic colorectal cancer and Lynch syndrome. Virchows Archiv. 2013 2013/11/01;463(5):613-21. 
20. Krypuy M, Newnham GM, Thomas DM, Conron M, Dobrovic A. High resolution melting analysis for the rapid and sensitive detection of mutations in clinical samples: KRAS codon 12 and 13 mutations in non-small cell lung cancer. BMC cancer. 2006 Dec 21;6:295. PubMed PMID: 17184525. Pubmed Central PMCID: PMC1769510. Epub 2006/12/23. eng.

21. Montgomery J, Wittwer CT, Palais R, Zhou L. Simultaneous mutation scanning and genotyping by high-resolution DNA melting analysis. Nature protocols. 2007;2(1):59-66. PubMed PMID: 17401339. Epub 2007/04/03. eng.

22. Simi L, Pratesi N, Vignoli M, Sestini R, Cianchi F, Valanzano R, et al. Highresolution melting analysis for rapid detection of KRAS, BRAF, and PIK3CA gene mutations in colorectal cancer. American journal of clinical pathology. 2008 Aug;130(2):247-53. PubMed PMID: 18628094. Epub 2008/07/17. eng.

23. Fadhil W, Kindle K, Jackson D, Zaitoun A, Lane N, Robins A, et al. DNA content analysis of colorectal cancer defines a distinct 'microsatellite and chromosome stable' group but does not predict response to radiotherapy. International Journal of Experimental Pathology. 2014 ;95(1):16-23. PubMed PMID: PMC3919646.

24. Fadhil W, Ibrahem S, Seth R, AbuAli G, Ragunath K, Kaye P, et al. The utility of diagnostic biopsy specimens for predictive molecular testing in colorectal cancer. Histopathology. 2012;61(6):1117-24.

25. Seth R, Crook S, Ibrahem S, Fadhil W, Jackson D, Ilyas M. Concomitant mutations and splice variants in $<$ em $>$ KRAS $</$ em $>$ and $<$ em $>$ BRAF $</$ em $>$ demonstrate complex perturbation of the Ras/Raf signalling pathway in advanced colorectal cancer. Gut. 2009;58(9):1234-41.

26. Ibrahem S, Seth R, O'Sullivan B, Fadhil W, Taniere P, Ilyas M. Comparative analysis of pyrosequencing and QMC-PCR in conjunction with high resolution melting for KRAS/BRAF mutation detection. International Journal of Experimental Pathology. 2010;91(6):500-5.

27. Fadhil W, Ibrahem S, Seth R, Ilyas M. Quick-multiplex-consensus (QMC)PCR followed by high-resolution melting: a simple and robust method for mutation detection in formalin-fixed paraffin-embedded tissue. Journal of Clinical Pathology. 2010 February 1, 2010;63(2):134-40. 
28. Ahmed D, Eide PW, Eilertsen IA, Danielsen SA, Eknas M, Hektoen M, et al. Epigenetic and genetic features of 24 colon cancer cell lines. Oncogenesis. 2013 09/16/online;2:e71.

29. Bacher JW, Flanagan LA, Smalley RL, Nassif NA, Burgart LJ, Halberg RB, et al. Development of a fluorescent multiplex assay for detection of MSI-High tumors. Disease markers. 2004;20(4-5):237-50. PubMed PMID: 15528789. Pubmed Central PMCID: PMC3839403. Epub 2004/11/06. eng.

30. Boland CR, Thibodeau SN, Hamilton SR, Sidransky D, Eshleman JR, Burt RW, et al. A National Cancer Institute Workshop on Microsatellite Instability for Cancer Detection and Familial Predisposition: Development of International Criteria for the Determination of Microsatellite Instability in Colorectal Cancer. Cancer Research. 1998;58(22):5248-57.

31. Dietmaier W, Hofstädter F. Detection of Microsatellite Instability by Real Time PCR and Hybridization Probe Melting Point Analysis. Laboratory Investigation. 2001 10/01/online;81:1453.

32. Suraweera N, Duval A, Reperant M, Vaury C, Furlan D, Leroy K, et al. Evaluation of tumor microsatellite instability using five quasimonomorphic mononucleotide repeats and pentaplex PCR. Gastroenterology. 2002 2002/12/01/;123(6):1804-11.

33. Janavicius R, Matiukaite D, Jakubauskas A, Griskevicius L. Microsatellite Instability Detection by High-Resolution Melting Analysis. Clinical Chemistry. 2010 November 1, 2010;56(11):1750-7.

34. Zhang L. Immunohistochemistry versus Microsatellite Instability Testing for Screening Colorectal Cancer Patients at Risk for Hereditary Nonpolyposis Colorectal Cancer Syndrome: Part II. The Utility of Microsatellite Instability Testing. The Journal of Molecular Diagnostics : JMD. 2008 04/28/accepted;10(4):301-7. PubMed PMID: PMC2438197.

35. Kondelin J, Tuupanen S, Gylfe AE, Aavikko M, Renkonen-Sinisalo L, Järvinen $\mathrm{H}$, et al. 3'-UTR poly(T/U) repeat of EWSR1 is altered in microsatellite unstable colorectal cancer with nearly perfect sensitivity. Familial Cancer. 2015;14(3):449-53.

36. Kishore S, Piscuoglio S, Kovac MB, Gylling A, Wenzel F, Trapani F, et al. 3'UTR Poly(T/U) Tract Deletions and Altered Expression of <em>EWSR1</em> Are a Hallmark of Mismatch Repair-Deficient Cancers. Cancer Research. 2014;74(1):22434. 
37. Woerner SM, Yuan YP, Benner A, Korff S, von Knebel Doeberitz M, Bork P. SelTarbase, a database of human mononucleotide-microsatellite mutations and their potential impact to tumorigenesis and immunology. Nucleic Acids Research. 2010;38(suppl_1):D682-D9.

38. Ebili HO, Hassall JC, Fadhil W, Ham-Karim H, Asiri A, Raposo TP, et al. "Squirrel" Primer-Based PCR Assay for Direct and Targeted Sanger Sequencing of Short Genomic Segments. Journal of biomolecular techniques : JBT. 2017 Sep;28(3):97-110. PubMed PMID: 28785174. Pubmed Central PMCID: PMC5460669. Epub 2017/08/09. eng.

39. Deng G, Chen A, Hong J, Chae HS, Kim YS. Methylation of CpG in a small region of the hMLH1 promoter invariably correlates with the absence of gene expression. Cancer Res. 1999 May 01;59(9):2029-33. PubMed PMID: 10232580. Epub 1999/05/08. eng.

40. Gausachs M, Mur P, Corral J, Pineda M, Gonzalez S, Benito L, et al. MLH1 promoter hypermethylation in the analytical algorithm of Lynch syndrome: a costeffectiveness study. European journal of human genetics : EJHG. 2012 Jul;20(7):762-8. PubMed PMID: 22274583. Pubmed Central PMCID: PMC3376264. Epub 2012/01/26. eng.

41. Stewart S, Wickramasinghe D, Dorrance AE, Robertson AE. Comparison of three microsatellite analysis methods for detecting genetic diversity in Phytophthora sojae (Stramenopila: Oomycete). Biotechnology Letters. 2011;33(11):2217-23. PubMed PMID: PMC3181407.

42. Klingbiel D, Saridaki Z, Roth AD, Bosman FT, Delorenzi M, Tejpar S. Prognosis of stage II and III colon cancer treated with adjuvant 5-fluorouracil or FOLFIRI in relation to microsatellite status: results of the PETACC-3 trial†. Annals of Oncology. 2015;26(1):126-32.

43. Des Guetz G, Schischmanoff O, Nicolas P, Perret GY, Morere JF, Uzzan B. Does microsatellite instability predict the efficacy of adjuvant chemotherapy in colorectal cancer? A systematic review with meta-analysis. European journal of cancer (Oxford, England : 1990). 2009 Jul;45(10):1890-6. PubMed PMID: 19427194. Epub 2009/05/12. eng.

44. Llosa NJ, Cruise M, Tam A, Wicks EC, Hechenbleikner EM, Taube JM, et al. The vigorous immune microenvironment of microsatellite instable colon cancer is 
balanced by multiple counter-inhibitory checkpoints. Cancer discovery. 2015 Jan;5(1):43-51. PubMed PMID: 25358689. Pubmed Central PMCID: Pmc4293246. Epub 2014/11/02. eng.

45. Xiao Y, Freeman GJ. The Microsatellite Instable Subset of Colorectal Cancer Is a Particularly Good Candidate for Checkpoint Blockade Immunotherapy. Cancer discovery. 2015 January 1, 2015;5(1):16-8.

46. Nowak JA, Yurgelun MB, Bruce JL, Rojas-Rudilla V, Hall DL, Shivdasani P, et al. Detection of Mismatch Repair Deficiency and Microsatellite Instability in Colorectal Adenocarcinoma by Targeted Next-Generation Sequencing. J Mol Diagn. 2017 Jan;19(1):84-91. PubMed PMID: 27863258. Pubmed Central PMCID: PMC5225299. Epub 2016/11/20. eng. 
Table 1.

Mononucleotide markers

\begin{tabular}{|c|c|c|c|c|c|c|c|c|c|}
\hline ID & BCAT25 & BAT25 & BAT26 & MYB & NEWSR1 & MSI/MSS & $\begin{array}{c}\text { MLH1 } \\
\text { promoter }\end{array}$ & BRAF & pLS \\
\hline 1 & $U$ & $\mathrm{~s}$ & $U$ & $U$ & $U$ & MSI & Non-Meth & WT & Yes \\
\hline 2 & $U$ & $U$ & $U$ & $E$ & $\mathrm{~F}$ & MSI & Meth & M & No \\
\hline 3 & $S$ & $S$ & $S$ & $S$ & $S$ & MSS & Non-Meth & WT & No \\
\hline 4 & U & U & U & U & U & MSI & Meth & M & No \\
\hline 5 & $S$ & $S$ & $S$ & $S$ & $S$ & MSS & Non-Meth & WT & No \\
\hline 6 & $S$ & $S$ & S & $S$ & $S$ & MSS & Non-Meth & $M$ & No \\
\hline 7 & $\mathrm{~s}$ & $S$ & $\mathrm{~s}$ & $\mathrm{~S}$ & $S$ & MSS & Non-Meth & WT & No \\
\hline 8 & $U$ & $U$ & $U$ & $U$ & $U$ & $\mathrm{MSI}$ & Meth & M & No \\
\hline 9 & $U$ & $U$ & $U$ & $U$ & $U$ & $\mathrm{MSI}$ & Meth & $M$ & No \\
\hline 10 & U & U & U & U & U & MSI & Non-Meth & WT & Yes \\
\hline 11 & U & $u$ & $U$ & U & $U$ & MSI & Non-Meth & WT & Yes \\
\hline 12 & S & $S$ & S & S & $S$ & MSS & Non-Meth & WT & No \\
\hline
\end{tabular}

\section{Original data}

\begin{tabular}{ccc}
\hline IHC & & BRAF \\
\hline dMMR & MSH6- & WT \\
dMMR & MLH1 - & M \\
pMMR & & WT \\
dMMR & MLH1 - & M \\
pMMR & & WT \\
pMMR & & M \\
pMMR & & WT \\
dMMR & MLH1- & M \\
dMMR & MLH1- & M \\
dMMR & MSH2- & N/A \\
dMMR & MSH2- & WT \\
PMMR & & WT \\
\hline
\end{tabular}




\section{FIGURE LEGENDS}

Figure 1: Limit of detection of MSI screening by HRM analysis and capillary electrophoresis. Cell line genomic DNA from HCT116 (MSI) was admixed with DNA from SW837 (MSS) to produce differing proportion of MSI/MSS DNA with calculated percentage MSI DNA as indicated. Figure $1 A$ shows the analysis by HRM for each marker. A known MSS DNA sample was used as an internal reference standard. Samples containing $6.25 \%$ MSI DNA clearly show different melting pattern as compared to the MSS sample. Figure 1B shows the analysis of the same samples using capillary electrophoresis which depends on fragment size analysis. MSI is indicated by novel alleles of the mononucleotide markers with different sizes compared to the normal sample. MSI could be called in samples containing $6.25 \%$ MSI DNA.

Figure 2: Sequencing of Region $C$ of the MLH1 promoter. Region C was identified and Sanger sequencing (following PCR of bisulphite modified DNA) was performed on 10 cases of MSI CRC showing loss of MLH1 expression and MSI and 10 cases of MSS CRCs. Figure 2A shows a chromatogram from an MSI CRC. The sequencing trace shows double peaks indicating $C$ and $T$ residues at all eight $C p G$ sites within this region (arrows). The position in relation to the transcription start site is also indicated. All tested MSI CRCs showed this pattern. Figure 2B is a chromatogram from an MSS CRC and shows a single peak (arrows) indicating a $T$ residue at all eight CpG sites. All tested MSS CRCs showed this pattern and the absence of any double peaks would suggest that there is no partial methylation in these tumours.

Figure 3: HRM analysis of Region C of the MLH1 promoter. In order to define the melting patterns of methylated / non-methylated Region C of the MLH1 promoter, PCR was performed on fully methylated or fully non-methylated control DNA following bisulphite modification. Figure $3 \mathrm{~A}$ is a derivative plot of the PCR products and shows that each condition (i.e. methylated or non-methylated) had a distinct melting peak. The melting temperature $(\mathrm{Tm})$ of the methylated peak (double arrow) was higher than that of the non-methylated peak (single arrow) reflecting the higher proportion of cytosine residues within the fully methylated samples. Figure 3B shows the melting pattern of tumour samples which are pMMR (single arrow) and dMMR due to loss of MLH1 expression (double arrow). The pMMR tumours gave a single 
non-methylated peak. The dMMR tumours gave a double peak representing a methylated peak (from the tumour cells) and non-methylated peak (from the stromal cells). All pMMR tumours tested gave a single peak and all dMMR tumours gave a double peak thereby reinforcing the data that this region does not have a state of partial methylation.

Figure 4: Utility of the N_LyST panel to screen for Lynch Syndrome. Twelve cases of CRC were randomized and tested using N_LyST. Melting plots are shown for BCAT25 (A), BAT25 (B), BAT26 (C), MYB (D), EWSR1 (E) and BRAF (F). Derivative plots are shown for Region $\mathrm{C}$ of $\mathrm{MLH1}(\mathrm{G})$. It can be clearly seen that the melt curves of the tumours with MSI are different from those with MSS. If any marker in a tumour with MSI is stable, it will usually melt in the same way as the markers in the MSS tumours. In D there is one case with MSI (asterisked) which melted differently from the rest of the MSS category but also clustered independently from the other six MSI cases, hence this was called equivocal. F shows that the melt curves of the tumours with wild type BRAF are different from those with mutant BRAF. G is a derivative plot and tumours demonstrate two discrete melting forms: "methylated" comprising two melting peaks which represent methylated DNA (from tumour epithelium) and non-methylated DNA (from tumour stroma) or "non-methylated" comprising one melting peak which characterises a completely non-methylated tumour and stroma cell population.

Table 1: Screening cancers for Lynch Syndrome using the N_LyST panel.

Twelve cancers from the Nottingham cohort were screened blind for Lynch Syndrome in a single PCR run (see also Figure 4). The left hand side of the table shows the N_LyST data whilst the original data for these cases are given on the right hand side of the table. The mononucleotide markers define whether a tumour has microsatellite instability (MSI) or whether it is microsatellite stable (MSS). The pattern of $\mathrm{MSI}$, non-methylated MLH1 promoter and wild-type BRAF is indicative of probable Lynch Syndrome whilst all other patterns indicate that Lynch Syndrome is not likely. From this series, three cases (shaded) were correctly identified as probable LS and nine as non-Lynch Syndrome (pLS = probably Lynch Syndrome, U=unstable, $S=$ stable, $\mathrm{E}=$ equivocal, $\mathrm{F}=$ failed, Non-Meth $=$ non-methylated, Meth = methylated, WT 
= wild type, $\mathrm{M}=$ mutant, $\mathrm{IHC}=$ immunohistochemistry, $\mathrm{dMMR}=$ deficient mismatch repair, $\mathrm{pMMR}=$ proficient mismatch repair, MLH1 $-=$ no MLH1 expression, MSH2 = no MSH2 expression, MSH6- = no MSH6 expression).

\section{Supplementary data}

Supplementary Figure 1: Mapping the $\mathrm{CpG}$ island of Region $\mathrm{C}$ of the MLH1 promotor. Using publicly available data, the $\mathrm{CpG}$ island of Region $\mathrm{C}$ (containing 8 $\mathrm{CpG}$ dinucleotides reported to be invariably completely methylated when MLH1 is silenced by promoter methylation. The $8 \mathrm{CpG}$ sites are underlined with green boxes and are located -46 to -111 upstream of the transcription start site (NCBI sequence ID: NC_018914.2). Primer attachment sites are highlighted in yellow.

Supplementary Table 1: Characteristics of genomic markers selected for MSI testing 
Supplementary Table 1: Characteristics of genomic markers selected for MSI testing

\begin{tabular}{|c|c|c|c|c|c|c|c|}
\hline Marker & $\begin{array}{l}\text { Genome } \\
\text { location }\end{array}$ & Gene/position & $\begin{array}{l}\text { Source of } \\
\text { marker* }\end{array}$ & $\begin{array}{c}\text { Published } \\
\text { mutation } \\
\text { rates }\end{array}$ & $\begin{array}{c}\text { Pcr } \\
\text { product } \\
\text { size }\end{array}$ & $\begin{array}{c}\text { Repeat } \\
\text { Size }\end{array}$ & $\begin{array}{l}\text { Primer Ta } \\
\text { range }\end{array}$ \\
\hline BCAT 25 & $3 p 22.1$ & CTNNB1/3'UTR & $\begin{array}{l}\text { Bioinformatic } \\
\text { analysis }\end{array}$ & Unknown & 100bp & T25 & $50-60^{\circ} \mathrm{C}$ \\
\hline BAT 25 & $4 q 12$ & CKIT/intron 13 & Literature & $96-100 \%$ & 85bp & $\mathrm{T} 25$ & $46-64^{\circ} \mathrm{C}$ \\
\hline BAT 26 & 2p21-p16.3 & MSH2/intron 5 & Literature & $92.5-100 \%$ & 77bp & A26 & $45-65^{\circ} \mathrm{C}$ \\
\hline EWSR1 & $22 q 12.2$ & EWSR1/3'UTR & Literature & $99.5 \%$ & $70 \mathrm{bp}$ & $\mathrm{T} 16$ & $45-60^{\circ} \mathrm{C}$ \\
\hline FBX046 & $19 q 13.32$ & FBXO46/3'UTR & www.seltarbase.org & $95-100 \%$ & 57bp & $\mathrm{T} 13$ & $45-65^{\circ} \mathrm{C}$ \\
\hline TP53(BAT34CA) & $17 p 13.1$ & TP53/3'UTR & www.seltarbase.org & $87.5 \%$ & 69bp & A18 & $47-65^{\circ} \mathrm{C}$ \\
\hline MYB & $6 q 23.3$ & MYB/intronic & www.seltarbase.org & $100 \%$ & 75bp & T23 & $47-65^{\circ} \mathrm{C}$ \\
\hline TYMS26 & $18 p 11.32$ & TYMS/5'UTR & $\begin{array}{c}\text { Bioinformatic } \\
\text { analysis }\end{array}$ & Unknown & $78 b p$ & A26 & $45-65^{\circ} \mathrm{C}$ \\
\hline
\end{tabular}


